\title{
Infinite limits in the iteration of entire functions
}

\author{
I. N. BAKER \\ Department of Mathematics, Imperial College of Science and Technology, \\ London SW7 2BZ, England
}

(Received 30 October 1987; revised 8 February 1988)

\begin{abstract}
If $f$ is a transcendental entire function and $D$ is a non-wandering component of the set of normality of the iterates of $f$ such that $f^{n} \rightarrow \infty$ in $D$ then $\log \left|f^{n}(z)\right|=\mathrm{O}(n)$ as $n \rightarrow \infty$ for $z$ in $D$. For a wandering component the convergence of $f^{n}$ to $\infty$ in $D$ may be arbitrarily fast.
\end{abstract}

\section{Introduction}

Denote by $f$ a non-linear entire function and set $N(f)=\left\{z ;\left(f^{n}\right)\right.$ is normal in some neighbourhood of $z\}$, where $f^{n}, n \in \mathbb{N}$, is the $n$th iterate of $f$. The complement of $N(f)$ in the plane is the Julia set $J(f)$. We recall that $J(f)$ is a non-empty perfect set and that $N(f)$ is completely invariant in the sense that $z$ belongs to $N(f)$ if and only if $f(z)$ belongs to $N(f)$ [6]. Thus each component $D$ of $N(f)$ is mapped by $f$ into some (possibly different) component of $N(f)$ : if each $f^{n}(D)$ belongs to a different component of $N(f)$ the component $D$ is called 'wandering'; if this is not the case there are integers $m \geq 0$ and $p \geq 1$ such that $f^{m}(D) \subset D_{1}$ where $D_{1}$ is a component of $N(f)$ such that $f^{p}\left(D_{1}\right) \subset D_{1}$.

We wish to study the ways in which $f^{n}(z)$ may tend to $\infty$ for $z \in N(f)$ as $n \rightarrow \infty$. There are special classes of functions, for example those transcendental $f$ such that all the singularities of $f^{-1}$ lie over a finite number of points, for which this is impossible [5]. In general, however, $f^{n}(z)$ may approach $\infty$ either for $z$ in a wandering component or for $z$ such that, for some $m \geq 0, f^{m}(z)$ belongs to an unbounded periodic component $D_{1}$ of $N(f)$ in which the iterates have limit $\infty$.

In situations where $f^{n}$ approaches a constant finite limit in a non-wandering component $D$ the classical theory (see e.g. [6]) gives simple asymptotic formulae for $f^{n}(z)$ as $n \rightarrow \infty$. It is perhaps surprising that something can be said even for infinite limits.

THEOREM 1. If $f$ is a transcendental entire function, if $z$ belongs to a non-wandering component of $N(f)$ and if $f^{n}(z) \rightarrow \infty$, then

$$
\log \left|f^{n}(z)\right|=\mathrm{O}(n), \quad(n \rightarrow \infty) .
$$

THEOREM 2. If $f$ is transcendental entire and $D$ is a component of $N(f)$ such that $f(D) \subset D$ and $f^{n} \rightarrow \infty$ in $D$, then there exist a curve $\Gamma$, which tends to $\infty$ in $D$, and 
positive constants $K, L$ such that $f(\Gamma) \subset \Gamma$ and

$$
K|z|<|f(z)|<L|z|, \quad z \text { in } \Gamma .
$$

THEOREM 3. If $z$ belongs to a wandering component of $N(f)$ then $\left|f^{n}(z)\right|$ may approach $\infty$ arbitrarily fast.

Theorem 1 does not hold for polynomials, where $O(n)$ becomes $O\left(K^{n}\right)$, for some $K>0$. The correctness of the orders of magnitude in Theorems 1 and 2 will be shown by a discussion of the example $f(z)=e^{-z}+k z, k>1$.

If it is known that $f$ has no wandering components, then Theorem 1 may be used to test whether certain points are in $J(f)$. It is also interesting that, according to a recent letter from A. Eremenko to the author, for any non-constant entire function $f$ there exist points $z$ (not necessarily belonging to $N(f)$ ) such that $f^{n}(z) \rightarrow \infty$.

Theorems 1 and 2 improve recent results in [3] and [9].

The paper is a more formal version of a lecture given at the Mathematical Institute of Academia Sinica, Beijing on 18th May 1987.

\section{Proof of Theorems 1 and 2}

It is sufficient to prove Theorem 1 for a component $D$ of $N(f)$ which is invariant in the sense that $f(D) \subset D$. Since $f^{n}(z) \rightarrow \infty$ for some $z$ in $D$ it then follows that $D$ is unbounded and that $f^{n} \rightarrow \infty$ locally uniformly in $D$. It was shown in [2] that any unbounded component of $N(f)$ is simply-connected. The desired result is thus contained in the following lemma.

Lemma 1. Suppose that $D$ is an unbounded simply-connected plane domain and that $g$ is a function which is analytic in $D$ and such that $g(D) \subset D, g^{n} \rightarrow \infty$ in $D$. Then for any $z \in D$ we have

Further, for any $z^{\prime} \in D$

$$
\log \left|g^{n}(z)\right|=O(n) \quad(n \rightarrow \infty) .
$$

$$
\log \left|g^{n}\left(z^{\prime}\right)\right|-\log \left|g^{n}(z)\right|=\mathrm{O}(1) \quad(n \rightarrow \infty) .
$$

Proof. Take a finite $\alpha \in \partial D$. Denote the hyperbolic distance of points $z, z^{\prime}$ of $D$ with respect to $D$ by $\left[z, z^{\prime}, D\right]$. Choose any point $z=z_{0}$ in $D$ and put $z_{n}=g^{n}\left(z_{0}\right), n \in \mathbb{N}$. Denote $H=\left[z_{0}, z_{1}, D\right]$ which is certainly positive since $g^{n}\left(z_{0}\right) \rightarrow \infty$. The map does not increase hyperbolic distance, so that $\left[z_{n}, z_{n+1}, D\right] \leq H$ for all $n$.

Note that if $\xi, \eta$ are in $D$ and $[\xi, \eta, D]=\delta$, then $|\xi-\alpha| \leq|\eta-\alpha| \cdot \exp (4 \pi \delta)$. For the function $w=\log (z-\alpha)$ maps $D$ onto a simply-connected domain $G$ which contains no vertical segment of length $>2 \pi$. Since any $w$ in $G$ has distance at most $\pi$ from $\partial G$, it follows from the Koebe distortion theorem as in e.g. [8, p. 6] that the Poincaré metric $\rho_{G}|d w|$ of $G$ satisfies

$$
\rho_{G}(w) \geq 1 /\{4 d(w, \partial G)\} \geq 1 /(4 \pi) .
$$

Thus if $\xi^{\prime}=\log (\xi-\alpha), \eta^{\prime}=\log (\eta-\alpha)$, we have

$$
\delta=[\xi, \eta, D]=\left[\xi^{\prime}, \eta^{\prime}, G\right] \geq(1 /(4 \pi)) \operatorname{Re}\left(\xi^{\prime}-\eta^{\prime}\right),
$$

which gives $|\xi-\alpha| \leq|\eta-\alpha| \exp (4 \pi \delta)$. 
In particular we have

$$
\left|z_{n+1}-\alpha\right|<K\left|z_{n}-\alpha\right|,
$$

where $K=\exp (4 \pi H)$ and so $\left|z_{n}-\alpha\right|<K^{n}\left|z_{0}-\alpha\right|$, which implies $\log \left|z_{n}\right|=\mathrm{O}(n)$ as $n \rightarrow \infty$.

Further if $z^{\prime}$ is another point of $D$ and $z_{n}^{\prime}=g^{n}\left(z^{\prime}\right)$ we put $\varepsilon=\left[z^{\prime}, z, D\right] \geq\left[z_{n}^{\prime}, z_{n}, D\right]$ and obtain

$$
L^{-1}<\left|\frac{z_{n}^{\prime}-\alpha}{z_{n}-\alpha}\right|<L, \quad L=\exp (4 \pi \varepsilon),
$$

which implies

$$
\log \left|z_{n}^{\prime}\right|-\log \left|z_{n}\right|=\mathrm{O}(1) \quad \text { as } n \rightarrow \infty .
$$

The result of Theorem 2 is contained in the slightly more general lemma:

LEMMA 2. If $g$ and $D$ satisfy the assumptions of Lemma 1 , then there exists a curve $\Gamma$, which tends to $\infty$ in $D$, and positive constants $K, L$, such that $g(\Gamma) \subset \Gamma$ and

$$
K|z|<|g(z)|<L|z|, \quad z \text { in } \Gamma .
$$

Proof. In the proof of the previous lemma we join $z_{0}$ to $z_{1}=g\left(z_{0}\right)$ by a path $\gamma$ in $D$ and change the constant $H$ to $H^{\prime}=\sup \left[z, z^{\prime}, D\right]$ for $z, z^{\prime}$ in $\gamma$. Then $\gamma_{n}=g^{n} \gamma$ joins $z_{n}$ to $z_{n+1}$ and $\Gamma=\bigcup \gamma_{n}$ is a path. For $z$ in $\gamma_{n}$ we have $\left[z, z_{n}, D\right] \leq H^{\prime},\left[z, z_{n+1}, D\right] \leq H^{\prime}$ and hence, by the argument of the previous lemma

$$
K^{-1}\left|z_{n}-\alpha\right|<|z-\alpha|<\min \left(K\left|z_{n}-\alpha\right|, K\left|z_{n+1}-\alpha\right|\right),
$$

where $K=\exp \left(4 \pi H^{\prime}\right)$. Thus $\Gamma \rightarrow \infty$. Moreover, for $z$ in $\gamma_{n}$ we have $g(z)$ in $\gamma_{n+1}$, so that

$$
|g(z)-\alpha| \leq K\left|z_{n+1}-\alpha\right| \leq K^{2}|z-\alpha|
$$

and similarly $|g(z)-\alpha| \geq K^{-2}|z-\alpha|$.

The proof is complete.

COROLlaRY. If in Lemma 2 we replace the assumption that $\mathrm{g}(D) \subset D$ by $\mathrm{g}^{p}(D) \subset D$ for some $p \in \mathbb{N}$, then we obtain a path $\Gamma$ on which $K|z|<\left|g^{p}(z)\right|<L|z|$. Assuming that $g$ is an entire function we can at least say that $g(z) \rightarrow \infty$ on $\Gamma$.

3. The example $f(z)=e^{-z}+k z, k>1$

For fixed $k>1$ there is an $x_{0}$ such that $k x-e^{-x}>x$ for $x \geq x_{0}$. If $H$ denotes the half plane $\left\{\operatorname{Re} z>x_{0}\right\}$ we have $f(H) \subset H$. Moreover it is clear that for real $x>x_{0}$, $f^{n}(x) \rightarrow \infty$. Thus $H$ is contained in a component $D$ of $N(f)$ such that $f^{n} \rightarrow \infty$ in $D, f(D) \subset D$. Further, $D$ must be simply-connected.

For real $x>x_{0}$ we have $x_{n}=f^{n}(x)>k^{n} x$ and $x_{n+1}-k x_{n}=\exp \left(-x_{n}\right)$. Thus, if $t_{n}=x_{n} / k^{n}$, then

$$
t_{n+1}-t_{n}<\left\{\exp \left(-x_{0} k^{n}\right)\right\} / k^{n+1},
$$

and so $\sum\left(t_{n+1}-t_{n}\right)<\infty$. Thus

$$
\lambda=\lim x_{n} k^{-n}
$$


is finite and

$$
\log \left(f^{n}(x)\right)=n \log k+\mathrm{O}(1) .
$$

By the final statement of Lemma 1 (1) also holds if $x$ is replaced by any $z$ in $D$.

\section{Proof of Theorem 3.}

In [1] some entire $f$ were constructed for which $N(f)$ has wandering components in which $f^{n}(z) \rightarrow \infty$ much faster than in Theorem 1. To obtain the result of Theorem 3 we modify a rather different argument in [4] to construct an example with the following properties.

Suppose that $10<a_{1}<a_{2}<\cdots$, where $a_{n}$ is a sequence with no other restriction except that $a_{n+1}-a_{n}>4$, so that $a_{n}$ may increase as fast as we please. Let $A_{m}=$ $\left\{z:\left|z-a_{m}\right| \leq 1\right\}$. Then there is an entire function $g$ such that $g\left(A_{m}\right) \subset A_{m+1}$, and each $A_{m}$ belongs to a different, simply-connected, wandering component of $N(g)$. $A_{m+1}$ lies entirely to the right of $\left\{\operatorname{Re} z=\frac{1}{2}\left(a_{m}+a_{m+1}\right)\right\}$.

First we recollect the following facts, also used in [4]. If $F$ denotes a closed subset of $\mathbb{C}$ and $C_{a}(F)$ the functions which are continuous on $F$ and analytic in $\stackrel{\circ}{F}$, then $F$ is called a Carleman set (for $\mathbb{C}$ ) if, for any $g$ in $C_{a}(F)$ and for any positive continuous function $\varepsilon$ on $F$, there is an entire function $f$ such that $|g(z)-f(z)|<\varepsilon(z)$, $z \in F$. By Arakelyan's theorem (e.g. [7, p. 137]) we have (i) $\hat{\mathbb{C}} \backslash F$ must be connected and also locally connected at $\infty$. If in addition to (i) we have (ii) for each compact $K$ the union $W(K)$ of those components of $\stackrel{\circ}{F}$ which meet $K$ is relatively compact in $\mathbb{C}$, then $F$ is indeed a Carleman set $([7$, p. 157]).

To construct the example introduce

$$
\begin{aligned}
L_{m} & =\left\{z: \operatorname{Re} z=\frac{1}{2}\left(a_{m}+a_{m+1}\right)\right\}, \quad m \in \mathbb{N}, \\
B & =\{z:|z+6| \leq 1\},
\end{aligned}
$$

and let $\delta, \delta_{m}$ be positive numbers so small that $|w-\pi i-\log 6|<\delta$ implies $\left|e^{w}+6\right|<\frac{1}{2}$, and $\left|w-\log a_{m+1}\right|<\delta_{m}$ implies $\left|e^{k^{\prime}}-a_{m+1}\right|<\frac{1}{2}$.

Since the set $F=B \cup\left\{\bigcup_{m}\left(A_{m} \cup L_{m}\right)\right\}$ is a Carleman set there exists an entire function $h$ such that

$$
\begin{array}{ll}
|h(z)-\pi i-\log 6|<\delta, & z \in L_{m}, \\
|h(z)-\pi i-\log 6|<\delta, & z \in B, \\
\left|h(z)-\log a_{m+1}\right|<\delta_{m+1}, & z \in A_{m} .
\end{array}
$$

Then $g=e^{h}$ is entire and satisfies $g\left(A_{m}\right) \subset A_{m+1}$, and so $g^{n} \rightarrow \infty$ in each $A_{m}$, uniformly. Thus $A_{m} \in N(g)$.

Now $g$ maps $B$ into $\left\{z ;|z+6|<\frac{1}{2}\right\}$, so that $B$ contains an attractive fixed point $\xi$ and $g^{n} \rightarrow \xi$ in $B$. Further $g\left(L_{m}\right) \subset B$ so that $g^{n} \rightarrow \xi$ in $L_{m}$ and each $L_{m}$ belongs to a component of $N(g)$ different from the $A_{m}$. Thus the $A_{m}$ are wandering components of $N(g)$, as is, indeed, apparent from the rate at which $g^{n} \rightarrow \infty$ in $A_{m}$.

Finally, since by construction $g^{n} \neq 0$, we have that the $1 / g^{n}$ are entire functions which converge to the limit 0 in $A_{m}$ and so in the whole component $D_{m}$ of $N(g)$ to which $A_{m}$ belongs. Hence $D_{m}$ is simply-connected. 


\section{REFERENCES}

[1] 1. N. Baker. An entire function which has wandering domains. I. Australian Math. Soc. 22 (Ser. A) (1976), 173-176.

[2] I. N. Baker. The domains of normality of an entire function. Ann. Acad. Sci. Fennicae AI Math. 1 (1975), 277-283.

[3] I. N. Baker. Iteration of polynomials and transcendental entire functions. J. Australian Math. Soc. (A) 30 (1981), 483-495.

[4] I. N. Baker. Wandering domains for maps of the punctured plane. Ann. Acad. Sci. Fennicae. A1, Math. 12 (1987), 191-198.

[5] A. Eremenko \& M. Lyubich. Iterations of entire functions (Russian). Dokl. Akad. Nauk SSSR 279

(1) (1984), 25-27 and preprint, Kharkov 1984.

[6] P. Fatou. Sur l'itération des fonctions transcendantes entières. Acta Math. 47 (1926), 337-370.

[7] D. Gaier. Lectures on Complex Approximation, Birkhäuser: Verlag: Basel-Boston-Berlin, 1987.

[8] O. Lehto. Univalent functions and Teichmüller spaces, Springer: New York, 1987.

[9] C. McMullen. Area and Hausdorff dimension of Julia sets of entire functions. Trans. Amer. Math. Soc. 300 (1) (1987), 329-342. 